\title{
Effectiveness of continuous veno-venous hemofiltration in the treatment of severe acute pancreatitis
}

\author{
XUAN CHEN ${ }^{1,2}$, MEIFANG SUN ${ }^{3}$, XIUHONG MAO $^{1}$, XIAOXIA LIU $^{1}$ and WEIHE SUN ${ }^{1}$ \\ ${ }^{1}$ Department of Intensive Care Unit, Affiliated Dongtai Hospital of Nantong University, Dongtai, Jiangsu 224200; \\ ${ }^{2}$ Department of Anesthesiology, The First Affiliated Hospital of Nanjing Medical University, Nanjing, Jiangsu 210029; \\ ${ }^{3}$ Department of Nursing, Affiliated Dongtai Hospital of Nantong University, Dongtai, Jiangsu 224200, P.R. China
}

Received June 11, 2018; Accepted January 30, 2019

DOI: $10.3892 /$ etm. 2019.7246

\begin{abstract}
Continuous veno-venous hemofiltration (CVVH) in the treatment of severe acute pancreatitis (SAP) was investigated. A total of 60 patients with SAP treated in Affiliated Dongtai Hospital of Nantong University from October 2015 to October 2017 were enrolled in this retrospective study. Among them, 32 patients received $\mathrm{CVVH}$ and routine internal medicine therapy (the CVVH group), and 28 received routine internal medicine therapy (the control group). Then biochemical indexes and severity of SAP after treatment were detected to investigate the roles of CVVH and routine internal medicine therapy in treating SAP. Inflammatory factors in both the CVVH and control groups were significantly decreased after treatment $(\mathrm{P}<0.05)$, and $\mathrm{CVVH}$ could eliminate more factors. Comparison of blood biochemical indexes between the CVVH and control groups were carried out. Significant improvement was found $(\mathrm{P}<0.05)$ after $\mathrm{CVVH}$ and routine internal medicine therapy. Besides, Acute Physiology and Chronic Health Evaluation (APACHE)-II grade was significantly decreased in the CVVH group compared with that in the control group after 7 days. CVVH therapy can effectively reduce the levels of inflammatory factors in SAP patients, improve their biochemical and physiological indicators, and have high clinical application value.
\end{abstract}

\section{Introduction}

Acute pancreatitis (AP) is a common emergency. AP patients suffer from inflammatory responses such as bleeding, edema and necrosis caused by pancreatic tissue digestion due to abnormally activated pancreatic trypsin resulting from various factors such as drinking, overeating, hyperlipidemia and cholelithiasis $(1,2)$. The clinical manifestations of AP patients

Correspondence to: Dr Weihe Sun, Department of Intensive Care Unit, Affiliated Dongtai Hospital of Nantong University, 002 Kangfu West Road, Dongtai, Jiangsu 224200, P.R. China E-mail: fmtiy341@163.com

Key words: severe acute pancreatitis, continuous veno-venous hemofiltration, inflammation factors are acute and persistent abdominal pains, accompanied with clinical symptoms including nausea, vomiting, fever and stop of flatus and defecation in most patients (3). Severe acute pancreatitis (SAP) is a severe systemic inflammatory response (SIR) disorder initiated by pancreatic autodigestion. Inflammatory cells are activated and release massive cytokines, and the resulting cytokine-level chain reaction is an important reason for the aggravation of SAP (4). These inflammatory mediators cause systemic inflammatory response syndrome (SIRS) that is a major cause of complications and deaths (5). Based on the notions of SIRS and multiple organ dysfunction syndrome (MODS) raised in the 1990s, people now have understood the relationship between SIRS and MODS. In other words, SIRS is the foundation, and MODS is the most serious consequence during the development of SIRS and also the most common cause of death in severe pancreatitis (6). Blocking the development of SIRS into MODS can significantly reduce the mortality rate (7). Therefore, hemofiltration (HF) begins to be considered as a method to remove inflammatory mediators as much as possible in clinical practice (8). HF technique is able to evidently improve the roles of monocytes in SAP patients, rebuild the internal stability of the body's immune system, and non-selectively eliminate a variety of pro-inflammatory factors at the same time (9). The technique may be able to control SIRS, thus maintaining hemodynamic stability and preventing MODS.

Continuous veno-venous hemofiltration $(\mathrm{CVVH})$ in the treatment of early SAP mainly targets pro-inflammatory cytokines causing SIR, which is conducive to downregulating SIR and restoring the balance between pro-inflammatory response and anti-inflammatory response (10). Some studies have shown that application of short-term HF in early SAP is proved to be effective in blocking SIR, reducing pancreatic necrosis and protecting organ function, and have put forward at the same time that for fulminant acute pancreatitis (FAP), a good measure further improving the prognosis is to perform continuous HF after emergency operative drainage $(11,12)$. Through the mechanisms of convection and adsorption, HF can not only directly remove amylase and urea nitrogen in the blood, but also selectively eliminate molecules with a diameter less than filter pore size in the plasma using a certain pore size filter with features of large filter area and high permeability. Previous sepsis studies have also confirmed that HF can filter 
out cytokines to effectively reduce the level of cytokines in the plasma, thus preventing the pancreatic autodigestion caused by the over-activation of amylase, which plays a role in the protection of pancreatic cells, solves the excessive inflammatory reaction due to pro-inflammatory cytokines in early SAP, and is capable of preventing the progression of the disease (13-15). In addition, continuous blood purification technique can significantly improve the roles of mononuclear cells in patients and rebuild the homeostasis of the body's immune system.

\section{Patients and methods}

A total of 60 patients with SAP treated in Affiliated Dongtai Hospital of Nantong University (Dongtai, China) from October 2015 to October 2017 were collected, including 32 patients treated with $\mathrm{CVVH}$ and routine internal medicine therapy (CVVH group), and 28 patients treated with routine internal medicine therapy (control group). This study was approved by the Ethics Committee of Affiliated Dongtai Hospital of Nantong University. Signed informed consents were obtained from the participants before the study. The general clinical data of patients are shown in Table I. Diagnostic criteria for AP: patients met any two of the following criteria: i) symptoms such as acute and sudden onset of upper abdominal pain, which was persistent, severe, and often accompanied with back radiating pain, were observed, ii) serum amylase value was $>3 x$ upper limit of normal, and/or serum lipase activity was $>3 \mathrm{x}$ upper limit of normal, and iii) imaging manifestations of AP such as pancreatic inflammation, pancreatic necrosis and extra-pancreatic complications were observed on computed tomography (CT) and/or magnetic resonance imaging (MRI). Diagnostic criteria for SAP: patients met the following three conditions at the same time: i) the symptoms, signs, and blood and imaging findings met diagnostic criteria for AP, ii) persistent organ failure [single organ (respiratory organ, circulatory organ and kidney) failure or MODS] lasted for $>48 \mathrm{~h}$, and iii) improved Marshall score (16) was $\geq 2$ points.

Inclusion criteria were: i) patients meeting diagnostic criteria for SAP, ii) patients with time from onset to admission $<72 \mathrm{~h}$, iii) patients aged over 18 years and below 70 years, with no sex limitation, and iv) patients suffer from the disease for the first time and never received treatment for AP.

Exclusion criteria were: i) patients died within $24 \mathrm{~h}$ after admission, ii) SAP patients with surgical indications such as pancreatic pseudocysts, iii) SAP patients after surgery, iv) patients with malignant tumor or complicated with severe circulatory, respiratory and renal function diseases, v) patients with continuous renal replacement contraindications, vi) patients with mental illness or poor compliance, and vii) patients with other acute abdominal diseases such as acute cholecystitis, acute appendicitis and acute gastrointestinal perforation as well as diseases including chronic pancreatitis and mild acute pancreatitis (AP).

\section{Methods}

Routine internal medicine therapy. Vital signs of patients were closely monitored, and patients were forbidden from drinking and eating and inhaled oxygen (oxygen saturation was $>95 \%$ ). Gastrointestinal decompression was performed according to the condition of patients. In the early stage,
Table I. General data of patients with severe acute pancreatitis (mean \pm standard deviation).

\begin{tabular}{lccc}
\hline Groups & $\begin{array}{c}\text { HEMO } \\
\text { group } \\
(\mathrm{n}=32)\end{array}$ & $\begin{array}{c}\text { Control } \\
\text { group } \\
(\mathrm{n}=28)\end{array}$ & P-value \\
\hline Male & 20 & 18 & 0.8861 \\
Female & 12 & 10 & \\
Age (years $)$ & $54.32 \pm 10.65$ & $58.94 \pm 9.02$ & 0.0772 \\
Onset time $(\mathrm{h})$ & $21.03 \pm 6.36$ & $24.01 \pm 8.20$ & 0.1189 \\
Fever & 30 & 27 & 0.6348 \\
Nausea & 28 & 27 & 0.2119 \\
Emesis & 26 & 26 & 0.1870 \\
Abdominal pain & 29 & 27 & 0.3686 \\
Abdominal distension & 29 & 27 & 0.3686 \\
Jaundice & 10 & 8 & 0.8213 \\
\hline
\end{tabular}

$\mathrm{P}>0.05$, there was no significant difference between the two groups.

controlled liquid resuscitation was adopted, omeprazole sodium for injection was intravenously injected to protect gastric mucosa and indirectly inhibit pancreatic enzyme secretion. Patients received parenteral nutrition firstly, and then enteral nutrition after $48-72 \mathrm{~h}$ by placing a nasointestinal tube, and the dose of enteral nutrition was adjusted according to the result feedback of examinations. Then, $0.3 \mathrm{mg}$ octreotide acetate injection $+250 \mathrm{ml}$ normal saline was injected at a rate of $0.025 \mathrm{mg} / \mathrm{h}$ to inhibit pancreatic enzyme secretion. After admission, 200,000 U ulinastatin $+250 \mathrm{ml}$ normal saline was intravenously injected in the early stage to inhibit the activity of pancreatic enzymes relating to the progression of SAP. Symptomatic and supportive treatment was carried out according to the condition of patients.

$C V V H$. After definitive diagnosis, patients received CVVH treatment every day in the early stage using a multifiltrate blood purifier (Fresenius SE \& Co., Bad Homburg, Germany) and an Ultraflux AV600S hemodialysis filter (Fresenius SE \& Co.). The effective membrane area was $1.4 \mathrm{~mm}^{2}$. A total of $1,500 \mathrm{ml}$ normal saline $+37,500 \mathrm{U}$ unfractionated heparin was used for pre-rinse for $30 \mathrm{~min}$, and hemorrhage treatment was conducted after completion of pre-rinse.

Determination of biochemical indexes and severity of SAP. Venous blood was collected from patients before treatment and at 7 day during treatment to detect inflammatory factors such as tumor necrosis factor- $\alpha$ (TNF- $\alpha)$, interleukin-1 $\beta$ (IL-1 $\beta$ ) and IL-6, blood routine indexes, liver and renal function as well as amylase in the blood and urine. Acute Physiology and Chronic Health Evaluation (APACHE)-II grade was used to assess the severity of SAP.

Statistical analysis. Statistical Product and Service Solutions (SPSS) 20.0 software (IBM Corp., Armonk, NY, USA) was used for statistical analysis of data. Homogeneity test for variance was applied for data meeting normal distribution, using independent-sample or paired-sample t-test. Comparison between multiple groups was done using one-way ANOVA test followed by post hoc test (Least Significant Difference). 


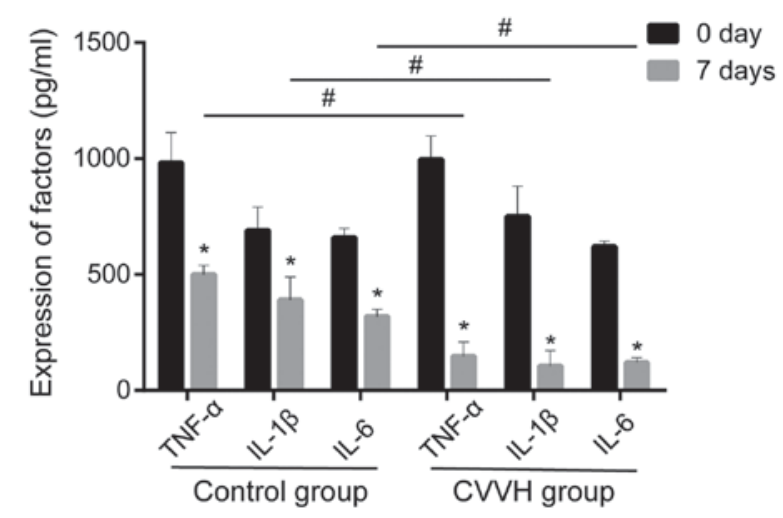

Figure 1. CVVH and routine internal medicine therapy inhibit the level of inflammatory factors. " $\mathrm{P}<0.05$, compared with before treatment. ${ }^{*} \mathrm{P}<0.05$, compared with the control group. $\mathrm{CVVH}$, continuous veno-venous hemofiltration.

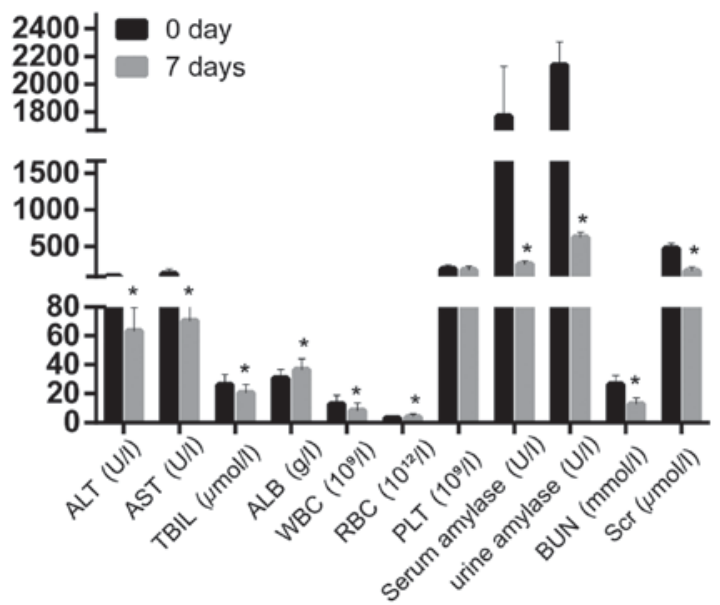

Figure 2. ALT, AST, TBIL, ALB, WBC, serum amylase, urine amylase, BUN and $\mathrm{Scr}$ are significantly lower after treatment compared with the level before treatment in control group. "P $<0.05$, compared with before treatment. ALT, alanine aminotransferase; AST, aspartate aminotransferase; TBIL, total bilirubin; ALB, albumin; WBC, white blood cell; RBC, red blood cell; PLT, platelets; BUN, blood urea nitrogen; Scr, creatinine.

Rank sum test was used for data not meeting normal distribution. Quantitative data were expressed as rate or percentage, using $\chi^{2}$ test, and $\alpha=0.05$ was used as the test level. $\mathrm{P}<0.05$ was considered to indicate a statistically significant difference.

\section{Results}

Inflammatory factors are significantly decreased in both CVVH and control groups after treatment. After analyzing the levels of TNF- $\alpha$, IL- $1 \beta$ and IL- 6 between the CVVH and control groups after treatment, it was found that these inflammatory factors were remarkably lower in the CVVH and control groups. Besides, comparison of expression of inflammatory factors between the two groups showed that HF and routine internal medicine therapy could eliminate more factors than the control group (Fig. 1).

Blood biochemical indexes after treatment in control group. Biochemical indexes such as alanine aminotransferase (ALT), aspartate aminotransferase (AST), total bilirubin (TBIL),

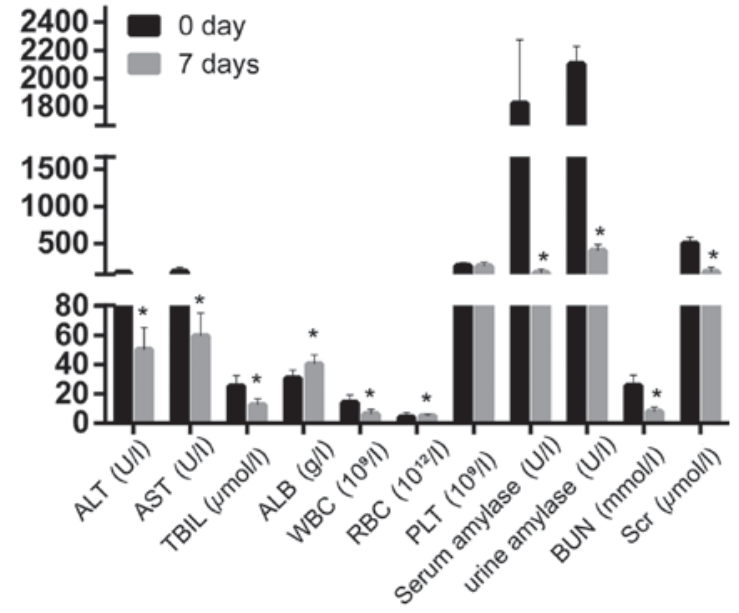

Figure 3. ALT, AST, TBIL, ALB, WBC, serum amylase, urine amylase, BUN and $\mathrm{Scr}$ are significantly decreased after $\mathrm{CVVH}$ treatment. " $\mathrm{P}<0.05$, compared with before treatment. ALT, alanine aminotransferase; AST, aspartate aminotransferase; TBIL, total bilirubin; ALB, albumin; WBC, white blood cell; RBC, red blood cell; PLT, platelets; BUN, blood urea nitrogen; Scr, creatinine; $\mathrm{CVVH}$, continuous veno-venous hemofiltration.

Table II. Comparison of biochemical index (mean \pm SD) between the two groups after 7-day treatment.

\begin{tabular}{lcccr}
\hline $\begin{array}{l}\text { Biochemical } \\
\text { index }\end{array}$ & $\begin{array}{c}\text { Control } \\
\text { group }\end{array}$ & $\begin{array}{c}\text { HEMO } \\
\text { group }\end{array}$ & t value & P-value \\
\hline ALT & $63.32 \pm 16.36$ & $50.22 \pm 15.22$ & 3.196 & 0.0023 \\
AST & $70.56 \pm 16.39$ & $59.36 \pm 15.88$ & 2.679 & 0.0096 \\
TBIL & $20.69 \pm 5.78$ & $12.36 \pm 4.25$ & 6.281 & $<0.0001$ \\
ALB & $36.58 \pm 7.55$ & $40.35 \pm 6.36$ & 2.075 & 0.0424 \\
WBC & $8.46 \pm 5.11$ & $6.02 \pm 3.21$ & 2.177 & 0.0335 \\
RBC & $3.98 \pm 2.12$ & $4.88 \pm 1.09$ & 2.203 & 0.0477 \\
PLT & $178.66 \pm 50.63$ & $190.88 \pm 56.21$ & 0.886 & 0.3793 \\
Serum & $256.98 \pm 45.32$ & $102.23 \pm 55.36$ & 11.90 & $<0.0001$ \\
amylase & & & & \\
Urine & $626.36 \pm 69.89$ & $400.56 \pm 85.96$ & 11.22 & $<0.0001$ \\
amylase & & & & \\
BUN & $12.88 \pm 4.21$ & $7.65 \pm 3.26$ & 5.322 & $<0.0001$ \\
Scr & $165.23 \pm 56.44$ & $120.36 \pm 57.01$ & 3.058 & 0.0034 \\
\hline
\end{tabular}

$\mathrm{P}<0.05$, there were significant differences in biochemical index between the two groups, and the clearance of CVVH group was better than that in the control group. ALT, alanine aminotransferase; AST, aspartate aminotransferase; TBIL, total bilirubin; ALB, albumin; WBC, white blood cell; RBC, red blood cell; PLT, platelets; BUN, blood urea nitrogen; Scr, creatinine; CVVH, continuous veno-venous hemofiltration.

albumin (ALB), white blood cell (WBC), serum amylase, urine amylase, blood urea nitrogen (BUN) and creatinine (Scr) were significantly lower after treatment compared with those before treatment, while red blood cells (RBC) were overtly higher than that before treatment, showing statistically significant differences $(\mathrm{P}<0.05)$. There was no statistically significant difference in platelets (PLT) before and after treatment ( $\mathrm{P}>0.05)$ (Fig. 2).

Blood biochemical indexes alter with HF and routine internal medicine therapy and CVVH group achieves better results 


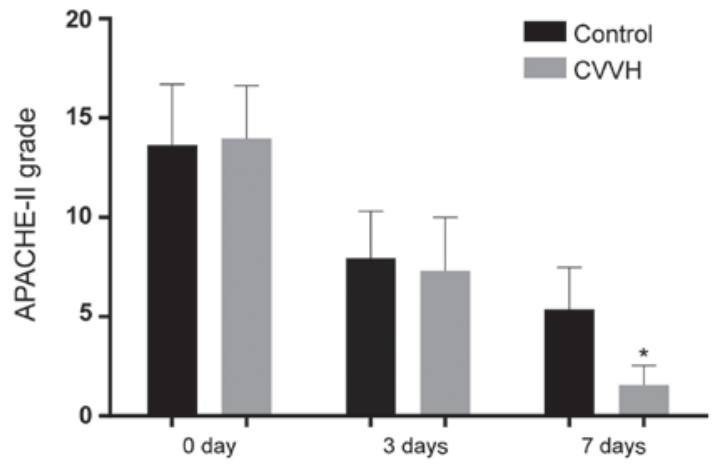

Figure 4. Significant differences after 7 days of treatment between the two groups. " $\mathrm{P}<0.05$, compared with the control group. APACHE, Acute Physiology and Chronic Health Evaluation; CVVH, continuous veno-venous hemofiltration.

than the control group. After CVVH treatment, ALT, AST, TBIL, ALB, WBC, serum amylase, urine amylase, BUN and Scr were significantly decreased (Fig. 3). Moreover, its clearance was better than that in the control group (Table II).

APACHE-II grade significantly decreases in the CVVH group compared with that in the control group after 7 days. There were no significant differences in APACHE-II grade between the two groups on the 1st day. APACHE-II was used to evaluate the severity of SAP at 3 and 7 days after treatment. The data suggested that there were no remarkable differences after 3 days of treatment. However, significant differences were detected after 7 days of treatment (Fig. 4).

\section{Discussion}

AP refers to pancreatic enzyme activation due to a variety of causes, with the main feature of local inflammatory response of the pancreas (17). Patients in severe cases may have SIRS and may be complicated with diseases of organ dysfunction. A large number of inflammatory cytokines produced during SAP can lead to damage of vascular endothelial cells and the activation of tissue factor-mediated abnormal coagulation, resulting in coagulation disorders, clinically manifested as thrombosis and secondary hemorrhage (18). Pathophysiological changes including micro-thrombosis formation and vascular permeability increase can lead to microcirculation disturbance, tissue edema and ischemia-hypoxia, which is one of the important mechanisms leading to MODS. Approximately $70 \%$ of SAP patients are complicated with acute respiratory distress syndrome ARDS), which is one of the major causes of death in SAP patients. For the treatment of SAP, the principle of 'early treatment and operation expansion' was followed in the early stage. However, in recent years, more attention has been paid to the coexistence of 'surgical treatment' and 'non-surgical' treatment in the patient-oriented personalized treatment program. Moreover, some researchers have proposed to determine the treatment plan according to the stage of the disease. At present, comprehensive treatment methods combining multiple ways have been formed. In the early stage of SAP (19), the therapeutic goal is to control further response of inflammation because of the possible occurrence of abdominal compartment syndrome, and the main treatment portions are the observation in the Intensive Care Unit (ICU) and the application of professional life-sustaining equipment in ICU for humoral recovery (20). In addition, vital signs and metabolic changes were observed in real time, and timely interventions, symptomatic treatment and strict control of complications were carried out. In ICU treatment for SAP, combined therapy should be performed based on the specific symptoms of different patients. Commonly used comprehensive treatment methods include nutritional support, blood purification, artificial respirator assisted ventilation and operative drainage.

$\mathrm{CVVH}$ is a blood purification technique that removes solutes by using convection (21). It was first used clinically in 1988 for acute and chronic renal failure patients who could not receive hemodialysis because they were complicated with hemodynamic instability or other complications, or used as an emergency renal replacement therapy. CVVH does not require complicated and expensive equipment. As a safe and effective method for blood purification, CVVH has been widely used in the rescue of critically ill patients. Since the filter has a molecular weight cut-off of $50 \mathrm{ku}$ and is mainly used for the removal of serum molecular weight substances, various major inflammatory cytokines can be removed via CVVH. The results of this study showed that there were obvious differences in clearance of inflammatory cytokines and improvement of blood biochemical markers and clinical symptoms between the CVVH and control groups $(\mathrm{P}<0.05)$.

In conclusion, our results clarified that $\mathrm{CVVH}$ therapy can decrease the expression of inflammatory factors, improve biochemical and physiological indicators in SAP patients, and thus needs to be promoted extensively.

\section{Acknowledgements}

Not applicable.

\section{Funding}

This work was supported by the Medical and Health Science and Technology Program of Yancheng city (YK2015082).

\section{Availability of data and materials}

All data generated or analyzed during this study are included in this published article.

\section{Authors' contributions}

$\mathrm{XC}$ and WS designed the study and performed the experiments. XC and MS collected the data. XM and XL analyzed the data. XC and WS prepared the manuscript. All authors read and approved the final manuscript.

\section{Ethics approval and consent to participate}

This study was approved by the Ethics Committee of Affiliated Dongtai Hospital of Nantong University (Dongtai, China). Signed informed consents were obtained from the patients or guardians.

\section{Patient consent for publication}

Not applicable. 


\section{Competing interests}

The authors declare that they have no competing interests.

\section{References}

1. Norman J: The role of cytokines in the pathogenesis of acute pancreatitis. Am J Surg 175: 76-83, 1998.

2. Jin Z, Xu L, Wang X and Yang D: Risk factors for worsening of acute pancreatitis in patients admitted with mild acute pancreatitis. Med Sci Monit 23: 1026-1032, 2017.

3. Hackert T, Hartwig W, Fritz S, Schneider L, Strobel O and Werner J: Ischemic acute pancreatitis: Clinical features of 11 patients and review of the literature. Am J Surg 197: 450-454, 2009.

4. Kashyap AS, Anand KP and Kashyap S: Severe acute pancreatitis. JAMA 292: 1305, 2004.

5. Kellum JA, Johnson JP, Kramer D, Palevsky P, Brady JJ and Pinsky MR: Diffusive vs. convective therapy: Effects on mediators of inflammation in patient with severe systemic inflammatory response syndrome. Crit Care Med 26: 1995-2000, 1998.

6. Beal AL and Cerra FB: Multiple organ failure syndrome in the 1990s. Systemic inflammatory response and organ dysfunction. JAMA 271: 226-233, 1994.

7. Marshall JC: SIRS and MODS: What is their relevance to the science and practice of intensive care? Shock 14: 586-589, 2000.

8. Teraoka S, Mineshima M, Hoshino T, Ishimori I, Kaneko I, Sato Y, Haruguchi $\mathrm{H}$ and Agishi T: Can cytokines be removed by hemofiltration or hemoadsorption? ASAIO J 46: 448-451, 2000.

9. Gong D, Zhang P, Ji D, Chen Z, Li W, Li J, Li L and Liu Z: Improvement of immune dysfunction in patients with severe acute pancreatitis by high-volume hemofiltration: A preliminary report. Int J Artif Organs 33: 22-29, 2010.

10. Wang S, Xu L, Feng X, Li S, Feng Q, Liu C, Zhang X and Zhao Q: Is continuous venovenous hemofiltration effective against severe acute pancreatitis? Artif Organs 37: 615-622, 2013.

11. Yu C, Liu ZH, Chen ZH, Gong DH, Ji DX and Li LS: Improvement of monocyte function and immune homeostasis by high volume continuous venovenous hemofiltration in patients with severe acute pancreatitis. Int J Artif Organs 31: 882-890, 2008.
12. Xu J, Tian X, Zhang C, Wang M and Li Y: Management of abdominal compartment syndrome in severe acute pancreatitis patients with early continuous veno-venous hemofiltration. Hepatogastroenterology 60: 1749-1752, 2013.

13. Tang Y, Zhang L, Fu P, Kang Y and Liu F: Hemoperfusion plus continuous veno-venous hemofiltration in a pregnant woman with severe acute pancreatitis: A case report. Int Urol Nephrol 44: 987-990, 2012.

14. Jiang HL, Xue WJ, Li DQ, Yin AP, Xin X, Li CM and Gao JL: Influence of continuous veno-venous hemofiltration on the course of acute pancreatitis. World J Gastroenterol 11: 4815-4821, 2005.

15. Bellomo R, Tipping P and Boyce N: Continuous veno-venous hemofiltration with dialysis removes cytokines from the circulation of septic patients. Crit Care Med 21: 522-526, 1993.

16. Banks PA, Bollen TL, Dervenis C, Gooszen HG, Johnson CD, Sarr MG, Tsiotos GG and Vege SS; Acute Pancreatitis Classification Working Group: Classification of acute pancreatitis - 2012: Revision of the Atlanta classification and definitions by international consensus. Gut 62: 102-111, 2013.

17. Heath DI, Cruickshank A, Gudgeon AM, Jehanli A, Shenkin A and Imrie CW: The relationship between pancreatic enzyme release and activation and the acute-phase protein response in patients with acute pancreatitis. Pancreas 10: 347-353, 1995.

18. Yang R, Tenhunen J and Tonnessen TI: HMGB1 and histones play a significant role in inducing systemic inflammation and multiple organ dysfunctions in severe acute pancreatitis. Int J Inflamm 2017: 1817564, 2017.

19. Li HG, Zhou ZG, Li Y, Zheng XL, Lei S, Zhu L and Wang Y: Alterations of Toll-like receptor 4 expression on peripheral blood monocytes during the early stage of human acute pancreatitis. Dig Dis Sci 52: 1973-1978, 2007.

20. Bumbasirevic V, Radenkovic D, Jankovic Z, Karamarkovic A, Jovanovic B, Milic N, Palibrk I and Ivancevic N: Severe acute pancreatitis: Overall and early versus late mortality in intensive care units. Pancreas 38: 122-125, 2009.

21. Tan HK, Baldwin I and Bellomo R: Continuous veno-venous hemofiltration without anticoagulation in high-risk patients. Intensive Care Med 26: 1652-1657, 2000.

c) (7) $\Theta$ This work is licensed under a Creative Commons

Attribution-NonCommercial-NoDerivatives 4.0 International (CC BY-NC-ND 4.0) License. 